\title{
Associação entre níveis séricos de vitamina $D$ e componentes da síndrome metabólica em pacientes atendidos no centro de estudos e atendimento dietoterápico da Universidade do Estado da Bahia
}

\author{
Association between serum levels of vitamin D and components of the metabolic \\ syndrome in patients attended at the Center for Studies and Diet Therapy at the State \\ University of Bahia
}

Claubert Radamés Oliveira Coutinho-Lima ${ }^{1}$; Luama Santos Araújoㄹ; Denise Carneiro Lemaire ${ }^{2 *}$; Domingos Lázaro Rios ${ }^{3}$; Gildasio Carvalho da Conceicao4; Najara Amaral Brandão5; Edilene Maria Queiroz Araújo ${ }^{6}$

${ }^{1}$ Doutorando em Processos Interativos dos Órgãos e Sistemas, UFBA.; ${ }^{2}$ Doutorado em Doctorat En Immunologie - Université Daix Marseille II. Professor Pleno da Universidade do Estado da Bahia.; ${ }^{3}$ Doutorado em Genética e Biologia Molecular pela UFBA.; ${ }^{4}$ Especialista em Análises Clínicas e Genética Bioquímica.; ${ }^{5}$ Nutricionista.; ${ }^{6}$ Doutora em Biotecnologia e Genética pela Universidade Estadual de Feira de Santana.

\begin{abstract}
Resumo
Introdução: a síndrome metabólica é um conjunto de disfunções que ocorre no metabolismo. Por sua vez, as disfunções endocrinometabólicas, incluindo componentes da síndrome, tais como obesidade, diabetes mellitus, elevação da pressão arterial e dislipidemias, têm sido associadas a baixos níveis séricos de vitamina D. A Hipovitaminose D tem atingido altas prevalências em diferentes populações mundiais, sobretudo em portadores desta síndrome. Metodologia: estudo observacional analítico, descritivo, de corte transversal. Os pacientes foram diagnosticados com síndrome metabólica a partir das recomendações da IDF, 2006. A coleta de dados foi realizada no período de agosto de 2015 a julho de 2016 e para determinação da concentração sérica de 25-hidroxivitamina $D$ foi utilizado o método de quimioluminescência. Resultados: a hipovitaminose $D$ foi observada em $77,6 \%$ dos pacientes. Dentre os componentes da SM a hipertensão arterial foi o mais prevalente. A análise de dados evidenciou correlação inversamente proporcional entre os níveis séricos de $25(\mathrm{OH}) \mathrm{D}$, com as medidas de circunferência da cintura $(r=-0,245$; pvalor $=0,013)$, com níveis séricos de triglicerídeos $(r=-1,54$; pvalor $=0,030)$ e também com valores de Índice de Massa Corporal $(r=-0,225 ; p v a l o r=0,023)$. Conclusão: neste estudo foi observado alta prevalência de hipovitaminose $D$ e evidenciada correlação inversa entre os níveis séricos de vitamina D e as medidas de Circunferência da Cintura, de níveis séricos de triglicerídeos e valores de IMC.
\end{abstract}

Palavras-Chave: Vitamina D. Hipovitaminose D. Síndrome Metabólica.

\begin{abstract}
Introduction: metabolic syndrome is a condition in which the individual manifests a set of metabolic dysfunctions. Endocrine-metabolic dysfunctions, including components of the syndrome, such as diabetes mellitus, elevated blood pressure and dyslipidemias, have been associated with low serum levels of vitamin D. Hypovitaminosis D has reached high prevalence in different world populations, Patients with this syndrome. Metodology: descriptive, cross-sectional observational study. Patients were diagnosed with metabolic syndrome from the recommendations of the IDF, 2006. Data collection was performed from August 2015 to July 2016 and for determination of the serum concentration of 25-hydroxyvitamin D was used the chemiluminescence method. Results: hypovitaminosis D was observed in $77.6 \%$ of the patients. Among the components of MS, arterial hypertension was the most prevalent. Data analysis showed an inversely proportional correlation between $25(\mathrm{OH}) D$ serum levels and waist circumference $(r=-0.245$; pvalor $=0.013)$, with serum triglyceride levels $(r=-1.54$; pvalor $=0.030)$ and also with values of Body Mass Index $(r=-0.225$, pvalor $=0.023)$. Conclusion: in this study, a high prevalence of $D$-hypovitaminosis was observed and an inverse correlation was found between serum vitamin $D$ levels and Waist Circumference, serum triglyceride levels and BMI values.
\end{abstract}

Key words: Vitamin D. Hypovitaminosis D. Metabolic Syndrome.

\section{INTRODUÇÃO}

A síndrome metabólica (SM) é uma condição na qual o indivíduo manifesta um conjunto de disfunções no metabolismo que estão associados com o surgimento

Correspondente/Corresponding: * Denise Carneiro Lemaire - Instituto de Ciências da Sáude. Universidade Federal da Bahia - End.: Av. Reitor Miguel Calmon, s/n, Vale do Canela, Salvador - BA. CEP: 40110-100 - Tel: (71) 98737-2567 - E-mail:dc_lemaire@hotmail.com de doenças cardiovasculares (DCV) (BLACKFORD et al., 2016; KAUR, 2014). A SM, atinge entre 20 e 30\% da população mundial (MAXIMINO et al., 2015) e no Brasil prevalências ocorre entre 14,9 e 65,3\% (VIDIGAL et al., 2013). Contemporaneamente a SM é caracterizada pela presença de obesidade central, hipertensão arterial sistêmica, além de alteração do metabolismo glicídico e lipídico, como a elevação da glicemia de jejum, o aumento dos níveis séricos de triglicerídeos e redução de HDL-c (ABESO, 2013; I-DBSM, 2005). O seu diagnóstico, 
portanto, corresponde a junção de pelo menos três destes componentes descritos (AZAMBUJA et al., 2015).

Diversos autores têm sugerido que a presença de obesidade é um importante fator para o surgimento da SM (CASTRO et al., 2014) e tem sido associada a baixos níveis séricos de vitamina $D(V D)$, bem como seus componentes (SCHUCH; GARCIA; MARTINI, 2009; VERRUSIO et al., 2017; YIN et al., 2016). Por isso uma das justificativas para o aumento da hipovitaminose D (HV-D) em pacientes com

SM seria o acúmulo de tecido adiposo corporal, sobretudo na região abdominal. Muitos autores têm sugerido que o tecido adiposo atua como "sequestrador" da VD no organismo devido à alta afinidade existente entre o adipócito e esta vitamina lipossolúvel. Logo, devido a redução da biodisponibilidade desta vitamina no organismo, ocorre também a diminuição do estímulo a cascatas de reações hipotalâmicas que resulta no aumento da sensação da fome e na diminuição do gasto energético, o que pode potencializar o grau de obesidade e HV-D (SALEHPOUR et al., 2012; SANTOS et al., 2006; SILVA et al., 2008).

A deficiência e insuficiência de vitamina $D$, ou Hipovitaminose $D$, tem atingido altas prevalências em diferentes populações mundiais. A HV-D atinge cerca de 1 bilhão de pessoas em todo o mundo e tem sido caracterizada como problema importante para os profissionais e autoridades de saúde (BORGES; MARTINI; ROGERO, 2011; YIN et al., 2012). No Brasil, a elevada prevalência de HV-D tem sido observada em diversas regiões, principalmente em adultos e idosos e pode atingir mais de $90 \%$ dos indivíduos estudados. (SCHUCH; GARCIA; MARTINI, 2009).

Níveis sérios de vitamina $D$ pode ser influenciados por diversos fatores como a cor da pele, percentual de gordura, índice de massa corporal, localização geográfica (latitude), estação do ano e hábitos alimentares. Para Holick et al. (2011) a deficiência de vitamina D é uma pandemia muito comum na Austrália, Oriente Médio, Índia, África e América do Sul. No Brasil, a HV-D parece estar mais relacionada à baixa ingestão alimentar, a fatores genéticos e ao uso de medicamentos que diminuem a absorção desta vitamina, do que à exposição solar (SANTOS JUNIOR et al., 2011).

A principal forma de obtenção da VD é pela exposição à luz solar (80 a 90\%), mais especificamente a partir da radiação ultravioleta $\mathrm{B}(\mathrm{IOM}, 2011)$. Esta vitamina também pode ser obtida na alimentação (10 a $20 \%$ ) e, se necessário, com suplementação. As duas principais fontes dietéticas de VD são: D2 (ergocalciferol, de origem vegetal) e D3 (colecalciferol, de origem animal). Ambas as formas passam pelo sistema endocrinometabólico da vitamina D. Este sistema é formado por proteínas carreadoras e inúmeras enzimas (hepáticas e renais) responsáveis pela cascata de inativação e ativação até formar a $1 \alpha, 25$-diidroxi-vitamina $\left(1,25(\mathrm{OH})_{2} \mathrm{D}\right)$. Esta é a forma ativa da VD reconhecida como hormônio esteroide devido as suas amplas funções no organismo, como: controle da homeostase do cálcio e fósforo; modulação da autoimunidade e controle na síntese de interleucinas inflamatórias; controle da pressão arterial e do peso corporal (MAEDA et al., 2014).

Desta forma, os principais objetivos deste estudo foram: determinar a prevalência de hipovitaminose $D$ e dos componentes da SM e investigar possível associação entre ambos, em um grupo de pacientes com diagnóstico de SM, atendidos no Núcleo de Pesquisa e Extensão em Genômica Nutricional e Disfunções Metabólicas (GENUT) da Universidade do Estado da Bahia (UNEB), na cidade de Salvador - Bahia.

\section{METODOLOGIA}

Estudo observacional descritivo analítico, de cunho quantitativo, de corte transversal, inserido no projeto de pesquisa intitulado "Influência de dieta sem lactose sobre a síndrome metabólica: papel de polimorfimos nos genes da lactase, adiponectina e seu receptor, gip e seu receptor, TCF7L2, TNF, IL6 e NFKB"

$O$ atendimento clínico-nutricional foi realizado no Núcleo de Pesquisa e Extensão em Genômica Nutricional e Disfunções Metabólicas (GENUT) do Centro de Estudos e Atendimento Dietoterápico (CEAD), da UNEB, campus I, Salvador, Bahia.

População de estudo: A população de estudo foi composta de pacientes portadores de SM, de ambos os sexos, provenientes do Núcleo de Endocrinologia do Hospital Geral Roberto Santos (HGRS) ou por demanda espontânea. A SM foi diagnosticada a partir das recomendações propostas pela IDF, 2006, conforme descritas no quadro 1. Não foram incluídos no estudo portadores de: albinismo, síndrome de má absorção, doença hepática ou renal; pacientes em uso de anticonvulsivantes, glicocorticoides, imunossupressores ou antirretrovirais; suplementação de vitamina $D$ e aqueles que não assinaram o Termo de Consentimento Livre e Esclarecido (TCLE).

Quadro 1-Critérios de diagnóstico para a Síndrome Metabólica

\begin{tabular}{|l|l|}
\hline FATORES DE RISCO & CRITÉRIOS \\
\hline Circunferência da Cintura* & $\begin{array}{l}\geq 94 \mathrm{~cm} \text { em homens } \\
\geq 80 \mathrm{~cm} \text { em mulheres }\end{array}$ \\
\hline Pressão arterial* & $\geq 130 \times 85 \mathrm{mmHg} * *$ \\
\hline Glicemia de Jejum* & $\geq 100 \mathrm{mg} / \mathrm{dL}^{* *}$ \\
\hline Triglicerídeos* & $\geq 150 \mathrm{mg} / \mathrm{dL}^{* *}$ \\
\hline HDL-colesterol reduzido & $\begin{array}{l}<40 \mathrm{mg} / \mathrm{dL}^{*} \text { em homens** } \\
<50 \mathrm{mg} / \mathrm{dL}_{\text {mulheres }}^{* *}\end{array}$ \\
\hline
\end{tabular}

Fonte:Adaptado da IDF (2006) na qual a circunferência da cintura é um critério obrigatório.

*valores elevados; ** ou em tratamento medicamentoso.

Coleta de dados clínico-demográficos: a coleta de dados foi realizada no período de agosto de 2015 a julho de 2016, usando como instrumento uma ficha de anamnese, na qual constavam itens referentes a: dados sócio-demográficos; recordatório de 24 horas; frequência 
alimentar; história clínica, nutricional e familiar, além dos dados antropométricos. Cada um dos participantes foi entrevistado por pesquisadores treinados com a aplicação da anamnese.

Coleta de dados antropométricos: a identificação do Índice de Massa Corporal (IMC) foi realizada a partir da aferição do peso com o uso da balança digital e a altura com o auxílio do estadiômetro vertical. Uma fita inelástica foi utilizada para mensurar a circunferência da cintura que foi medida a partir do ponto médio entre o rebordo costal e a crista ilíaca, conforme descrito na I Diretriz Brasileira de Diagnóstico e Tratamento da Síndrome Metabólica, I-DBSM (2005). Para a aferição da pressão arterial sistêmica foi utilizado estetoscópio (da marca Littmann ${ }^{\circ}$ ) e tensiômetro (da marca $B i^{\circ}$ ) conforme as orientações preconizadas pela $\mathrm{VI}$ - Diretrizes da Sociedade Brasileira de Hipertensão - VI-DSBH, (2010).

Coleta de dados laboratoriais: Após jejum mínimo de $12 \mathrm{~h}$ foram coletadas amostras de sangue para realização das seguintes dosagens séricas: glicemia, triglicerídios, HDL-c (pelo método de colorimetria enzimática). Segundo a Sociedade Brasileira de Endocrinologia e MetabologiaSBEM (2014), a determinação da concentração sérica de 25-hidroxivitamina $D$ é o exame bioquímico mais indicado para avaliar o estado nutricional relativo aos níveis de VD e HV-D. Os valores de referência para tal diagnóstico utilizado nesta pesquisa estão apresentados no quadro 2 .

Quadro 2 - Valores de referência para o diagnóstico do estado nutricional relativo à vitamina $D$

\begin{tabular}{|l|l|l|}
\hline $\mathbf{2 5}(\mathbf{O H}) \mathbf{D}(\mathbf{n g} / \mathbf{m l})$ & $\mathbf{2 5}(\mathbf{O H}) \mathbf{D}(\mathbf{n m o l} / \mathbf{L})$ & Classificação \\
\hline$\leq 20$ & $\leq 50$ & Deficiência \\
\hline 21 a 29 & 51 a 74 & Insuficiência \\
\hline 30 a 100 & 75 a 250 & Suficiência \\
\hline$>100$ & $>250$ & Excesso / Tóxico \\
\hline
\end{tabular}

Fonte: Adaptado de Holick (2011) e Sociedade Brasileira de Endocrinologia e Metabologia (2014).

Foi utilizado o método de quimioluminescência para a dosagem da vitamina. A coleta de sangue e os exames laboratoriais foram realizadas no Laboratório da Associação de Pais e Alunos dos Excepcionais (APAE) da cidade de Salvador-BA.

Análise estatística: Os dados obtidos foram tabulados em planilha do Microsoft Excel versão 2013 e analisados com o auxílio do Software Pakckage for the Social Sciences (SPSS) versão 20.0 para Windows. Para a análise estatística dos dados coletados foi utilizado o método descritivo e para o teste de associação entre as variáveis foram utilizados os testes qui-quadrado e a correlação de Spearman.

Considerações éticas: Este projeto de pesquisa foi aprovado pela Comissão de Ética em Pesquisa da UNEB (CAAE:03409712.9.0000.0057) e obedeceu os critérios da Resolução 266, de 12 de dezembro de 2012, do Ministério da Saúde. Todos os pacientes assinaram o TCLE e estavam cientes de que poderiam desistir a qualquer tempo, sem que houvesse descontinuidade do atendimento nutricional.

\section{RESULTADOS E DISCUSSÃO}

A população em estudo foi composta de 205 voluntários portadores de síndrome metabólica, predominantemente do sexo feminino $82,9 \%$, e $17,1 \%$ do sexo masculino, com média de idade de 57 anos ( $28-82$ anos). A predominância do sexo feminino é também observada na população alvo, constituída de pacientes atendidos no CEAD/GENUT. Estes achados corroboram resultados de outros estudos que mostram que o fator sexo influencia a busca pelos serviços de saúde. Assim, ser do sexo feminino é um fator de proteção contra a não procura pelo serviço de saúde, enquanto ser do sexo masculino é um fator de risco. Há maior procura pelas mulheres aos serviços de saúde quando comparadas aos homens, fato que pode ser justificado por questões culturais e por existir uma maior preocupação do sexo feminino com a prevenção e/ ou acompanhamento de doenças (LEVORATO et al., 2014; PINHEIRO et al., 2002; TEIXEIRA, 2014).

Com relação aos cofatores da SM definidos pela IDF (2006), foi observada maior prevalência de hipertensão arterial ou elevação da pressão $(96,5 \%)$ (tabela 1$)$. A hipertensão arterial é considerada a doença crônica não transmissível mais comum no Brasil além de ser mais prevalente em indivíduos de cor de pele negra que em indivíduos brancos (ANDRADE et al., 2015; LOTUFO; BENSENOR, 2013). Em um estudo transversal, realizado em Goiânia, com 133 idosos portadores de SM, a elevação da pressão arterial também foi o cofator mais prevalente dentre os componentes da SM (VIEIRA; PEIXOTO; SILVEIRA, 2014).

Tabela 1 - Prevalência de componentes da síndrome metabólica na população de pacientes com diagnóstico de Síndrome Metabólica atendidos no Centro de Estudos e Atendimento Dietoterápico da UNEB

\begin{tabular}{lcc}
\hline COMPONENTES & $\mathbf{n}$ & \% \\
\hline Circunferência da Cintura* & 205 & 100 \\
Hipertensão arterial / PA & 193 & 96,5 \\
Hiperglicemia / DM & 172 & 84,3 \\
HDL-c reduzido & 204 & - \\
Mulheres & 115 & 67,6 \\
Homens & 21 & 60 \\
Triglicerídeos elevados & 148 & 72,5 \\
\hline
\end{tabular}

*Conforme critério adotado para definição da SM, 100\% dos participantes possuíam circunferência da cintura elevada; PA: pressão arterial; DM: Diabetes Mellitus;

Fonte: Autoria própria

A frequência observada do cofator hiperglicemia foi $84,3 \%$ (tabela 1). Araújo (2011) verificou dados semeIhantes ao deste estudo em uma pesquisa de prevalência da SM, diagnosticada também pela IDF (2006), em 20 
indivíduos atendidos em um Centro médico, no interior da Bahia. A hiperglicemia foi encontrada em $80 \%$ dos participantes como segundo cofator mais presente, após a hipertensão, o mesmo observado neste estudo. Indivíduos que apresentam SM possuem risco muito mais elevado para o desenvolvimento de diabetes tipo 2 (DM2) do que aqueles sem síndrome (KAUR, 2014). Os fatores de risco para diabetes mellitus tipo 2 incluem etnia, antecedentes familiares, obesidade, níveis séricos de HDL-colesterol reduzido e triglicerídeos elevados (MARINHO et al., 2013), situações encontradas na SM. Almeida et al. 2011, estudaram a prevalência de fatores associados ao DM2 em 299 indivíduos adultos da cidade de Fortaleza, CE. O estudo concluiu que $84 \%$ dos indivíduos apresentaram circunferência abdominal elevada, 83,3\% eram sedentários e $57,9 \%$ possuíam excesso de peso, comprovando a alta prevalência de condições de risco que podem levar ao diabetes, também presentes na SM.

A prevalência dos fatores componentes do perfil lipídico, como os triglicerídeos elevados foi de $72,5 \%$ e a HDL-colesterol reduzido foi de $67,6 \%$ nas mulheres e $60 \%$ nos homens, conforme mostrado na tabela 1 . A presença de dislipidemias em indivíduos com ou sem síndrome têm sido, geralmente, associado a condições que aumentam o risco de desenvolver doenças cardiovasculares e elevação do risco de outras complicações metabólicas. A HDL-c tem sido apontada como uma lipoproteína de difícil controle, visto que sua alteração envolve fatores genéticos e hábitos de vida (CARVALHO et al., 2015; MALTA et al., 2014). Em um estudo realizado com 378 idosos na região sul do Brasil, os autores identificaram dados semelhantes aos desta pesquisa para a prevalência de hipertrigliceridemia, $72 \%$, e redução de $\mathrm{HDL}-\mathrm{c}, 56 \%$ dos homens e $72 \%$ as mulheres (RIGO et al., 2009).

O aumento circulante de triglicerídeos e redução de HDL-c nos pacientes com SM pode ter início e ser acentuado devido a presença da obesidade, pois nesta condição há hiperplasia e hipertrofia dos adipócitos que passam a liberar mais ácidos graxos livres circulantes. Paralelo a este processo, ocorre o aumento da lipólise, que promove a liberação de triglicerídeos hepáticos e consequentemente, resulta em hipertrigliceridemia e diminuição da secreção de HDL colesterol (FERRARI, 2007; POZZAN et al., 2004).

Quanto a dosagem de 25(OH)D, foi observado níveis normais/suficientes em apenas $22,4 \%$ dos pacientes e maior prevalência de hipovitaminose $D$ em $77,6 \%$ dos indivíduos (tabela 2). Dentre os pacientes com hipovitaminose $D, 73 \%$ apresentaram valores insuficientes e $27 \%$ tiveram deficiência, ou seja, níveis ainda mais críticos. Nenhum paciente apresentou valores séricos acima da recomendação. $\mathrm{O}$ quadro de hipovitaminose D nos pacientes portadores de SM tem sido observado por diversos estudos em todo o mundo. (GODALA et al., 2016; KHOSRAVI-BOROUJENI; AHMED; SARRAFZADEGAN, 2016; LEE et al., 2016).
Tabela 2 - Níveis séricos de vitamina D 25(OH)D na população de pacientes com diagnóstico de Síndrome Metabólica atendidos no Centro de Estudos e Atendimento Dietoterápico da UNEB

\begin{tabular}{llll}
\hline Classificação & Níveis & (n) & (\%) \\
\hline NORMAL & Suficientes & 46 & 22,4 \\
HIPOVITAMINOSE D & Insuficientes & 116 & 73 \\
& Deficientes & 43 & 27 \\
\hline
\end{tabular}

Fonte: Autoria própria

As principais explicações para não captação e absorção desta vitamina pelo organismos envolve: doenças que alteram o metabolismo lipídico e que podem prejudicar a absorção; o sequestro de vitamina $D$ pelo tecido adiposo devido à sua estrutura e afinidade lipofílica; a baixa ingestão de alimentos fontes desta vitamina devido ao preço mais elevado desses produtos; sobretudo pelo fato da população do estudo ser composta, em sua maioria, por indivíduos de baixa renda e baixa exposição à radiação solar (GODALA et al., 2016; KHOSRAVI-BOROUJENI; AHMED; SARRAFZADEGAN, 2016; LEE et al., 2016; LÉGER-GUIST'HAU et al., 2016).

Em um estudo realizado na cidade de Salvador-Ba por Santos, Araújo e Melo no ano de 2013 com a mesma população estudada, as autoras identificaram que a maioria dos indivíduos apresentaram boa exposição ao sol do tipo moderada $(90,9 \%)$. Este nível de exposição é considerado satisfatório para a conversão da vitamina $D$ na pele. Para tal, é sabido que questões como a estação do ano, raça e idade podem influenciar na conversão da vitamina $\mathrm{D}$ na pele e atuar como fator condicionante de aparecimento de hipovitaminose $\mathrm{D}$ em uma determinada população. Outros autores descrevem que a incidência das radiações solares está mais atenuada em condições de baixas temperaturas, logo a conversão da VD na pele pode ficar reduzida (HOLICK et al., 2011; SANTOS, 2011). $\mathrm{O}$ que tem sido observado com maior frequência é que indivíduos com a pele mais escura, por possuir uma proteção diferenciada aos raios solares, precisariam de duas vezes mais o tempo necessário de exposição ao sol do que os de pele clara para que haja a mesma conversão de vitamina D dérmica (HOLICK et al., 2011; PREMAOR, FURLANETTO, 2006; SANTOS, 2011; SANTOS JUNIOR et al., 2011; SILVA et al., 2008).

Neste estudo foi encontrado correlação inversamente proporcional entre os níveis séricos da vitamina $D, 25(\mathrm{OH})$ $D$, com medidas de circunferência da cintura $(r=-0,245$; pvalor $=0,013$ ), com níveis séricos de triglicerídeos ( $r=-$ 1,54; pvalor $=0,030$ ) e também com valores de Índice de Massa Corporal $(r=-0,225$; pvalor=0,023), quadro 3. Por outro lado, não foi identificada associação estatisticamente significante entre níveis séricos de vitamina $D$ e componentes da SM nos indivíduos estudados. 
Quadro 3 - Correlação entre os níveis séricos de vitamina D 25(OH)D e os componentes da Síndrome Metabólica em pacientes atendidos no Centro de Estudos e Atendimento Dietoterápico da UNEB.

\begin{tabular}{|l|c|c|c|c|c|c|c|}
\hline & \multicolumn{7}{|c|}{ Níveis séricos de Vitamina D 25(OH)D } \\
\hline & CC & PAS & PAD & DM & HDI & TG & IMC* \\
\hline Coeficiente de correlação & $-0,245$ & $-0,014$ & $-0,079$ & $-0,106$ & $-0,064$ & $-0,154$ & $-0,225$ \\
\hline Pvalor & 0,013 & 0,851 & 0,272 & 0,134 & 0,366 & 0,030 & 0,023 \\
\hline
\end{tabular}

CC: Circunferência da Cintura; PAS: pressão arterial sistólica; PAD: pressão arterial diastólica; DM: diabetes ou níveis de glicemia; TG: triglicerídeos. *Além dos componentes da SM também foi avaliado o índice de massa corporal (IMC) por ser um indicador de obesidade. Foi utilizado o teste de correlação de Spearman.

Fonte: Autoria própria

Miñambres et al. (2012) realizaram um estudo transversal com 343 indivíduos obesos ou com sobrepeso e avaliaram possível correlação com níveis séricos da VD. Menores valores séricos desta vitamina foi associado com o maior grau de obesidade, o mesmo encontrado nesta pesquisa (quadro 3), especialmente quando o índice de massa corporal era maior que $40 \mathrm{~kg} / \mathrm{m}^{2}$. Dentre os indivíduos obesos que também apresentavam SM estes possuíam níveis séricos mais baixos de 25(OH)D do que os pacientes sem SM. Os autores concluíram que a probabilidade de desenvolver hipovitaminose $D$ foi maior em pacientes com SM que nos pacientes sem SM, independentemente do grau de obesidade.

Em um outro estudo de corte transversal Kayaniyil et al. (2011) também verificaram associação inversa entre as concentrações séricas de 25(OH)D com as medidas de circunferência da cintura; com os níveis séricos de triglicerídeos (quadro 3), HDL-c e de insulina de jejum em pacientes com SM de uma amostra multiétnica. Em concordância com este estudo, Rafaelli et al. (2015) descreveram que há bastante relação entre os níveis séricos da vitamina $\mathrm{D}$ e o DM tanto na prevenção quanto no tratamento do DM2 e que a deficiência de $25(\mathrm{OH}) \mathrm{D}$ pode alterar a produção e a secreção da insulina. A deficiência dessa vitamina parece conduzir à intolerância à glicose devido a redução da secreção insulínica, contribuindo para o maior risco de desenvolvimento de DM2.

Neves et al. (2012) investigaram possíveis associações entre os níveis séricos de 25(OH)D e pressão arterial em 91 idosos hipertensos e concluirão que a prevalência da hipovitaminose $D$ foi elevada e associada com maiores níveis pressóricos. Além do mais, a concentração sérica da 25(OH)D correlacionou-se inversamente com a pressão arterial sistólica.

Neste contexto, a deficiência ou insuficiência de vitamina $D$ pode ser observada em diversas fases da vida e está associada ao aumento da incidência de várias condições clínicas crônicas não infecciosas, especialmente a SM e seus componentes (MAEDA et al., 2014).

\section{CONCLUSÃO}

Neste estudo foi observado alta prevalência de hipovitaminose $\mathrm{D}$ e de todos os componentes da SM com destaque para a hipertensão arterial. Foi identificada correlação inversa entre os níveis séricos de Vitamina D com as medidas da circunferência da cintura, com níveis de triglicerídeos e com os valores de IMC. Embora nenhuma outra associação entre vitamina $D$ e os componentes da SM tenha sido encontrada neste estudo, novas estratégias de intervenção alimentar deverão ser criadas para contribuir no controle da deficiência/insuficiência de vitamina $\mathrm{D}$ em portadores de SM, com o objetivo de diminuir a incidência de doenças metabólicas que tem sido associadas com a síndrome.

\section{AGRADECIMENTOS}

A toda equipe do GENUT (nutricionistas, psicólogas e estagiários), da APAE de Salvador-BA e do núcleo de endocrinologia do Hospital Geral Roberto Santos (HGRS) pela parceria.

\section{REFERÊNCIAS}

ASSOCIAÇÃO BRASILEIRA PARA O ESTUDO DA OBESIDADE E DA SÍNDROME METABÓLICA (ABESO). Diretrizes Brasileiras de Obesidade. ABESO: São Paulo, 2010. Disponível em <http://www.abeso.org.br/pdf/diretrizes_brasileiras_obesidade_2009_2010_1.pdf> Acesso em: Jan. 2013.

ALI, N. S. et al. Retrospective analysis of metabolic syndrome: prevalence and distribution in executive population in urban Pakistan. Hindawi Publishing Corporation. Int. J. Family Medic, Pakistan, p.407-414, July 2012.

ALMEIDA, V. C. F. et al. Occupation and risk factors for type 2 diabetes: a study with health workers. Rev Latino-Am Enfermagem, São Paulo, v.19, n.3, p. 476-484, May./June 2011.

ANDRADE, S. S. A. et al. Prevalência de hipertensão arterial autorreferida na população brasileira: análise da Pesquisa Nacional de Saúde, 2013. Epidemiol. Serv. Saúde, Brasília, v. 24, n. 2, p. 297-304, abr./jun. 2015

ARAÚJO, C. S. Síndrome Metabólica em pacientes de um centro de especialidades médicas do interior da bahia. Revista Brasileira de Obesidade Nutrição e Emagrecimento, São Paulo, v. 5, n. 29, p.153-159, out. 2011.

AZAMBUJA, C. R. et al. O Diagnóstico da síndrome metabólica analisada sob diferentes critérios de definição. Rev. Baiana de Saúde Pública, Rio Grande do Sul, v.39, n.3, p.482-496 jul./set. 2015.

BLACKFORD, K. et al. Home-based lifestyle intervention for rural adults improves metabolic syndrome parameters and cardiovascular risk factors: a randomised controlled trial. Prevent. Medic., Perth, v.89, p.15-22, 2016

BORGES, M. C.; MARTINE L. A.; ROGERO M. M. Current perspectives on vitamin $D$, imune system and chronic diseases. Nutrition, Bristol, $v$. 27, p. 399-404, 2011. 
CARVALHO, C. A. et al. Associação entre fatores de risco cardiovascular e indicadores antropométricos de obesidade em universitários de São Luís, Maranhão, Brasil. Ciênc. Saúde Coletiva, São Paulo, v. 20, n. 2, p: 479-490, dez. 2015.

CASTRO, A. V. B. et al. Obesity, insulin resistance and comorbidities Mechanisms of association. Arq. Bras. Endocrinol. Metabol. São Paulo, v. 58, n. 6, p. 600-610, Aug. 2014.

CASTRO, L. C. G. O sistema endocrinológico: vitamina D. Arq. Bras. Endocrinol. Metab., São Paulo, v. 21, n. 12, p. 55-88, nov. 2011.

FERRARI, C. K. B. Atualização: fisiopatologia e clínica da síndrome metabólica. ACM Arq. Catarin. Med., Santa Catarina, v. 36, n. 4, p. 90-96, 2007.

GODALA, M. et al. Estimation of plasma 25(OH)D vitamin deficiency in patients with metabolic syndrome. Pol. Merkur. Lekarski, Polônia, v. 40, n. 239, p. 288-291, Mar. 2016.

HOLICK, M. F. et al. Evaluation, treatment, and prevention of vitamin d deficiency: an endocrine society clinical practice guideline. J. Clin. Endocrinol. Metab., Oxford, v. 96, n. 7, p. 122-132, June 2011.

I DIRETRIZ BRASILEIRA DE DIAGNÓSTICO E TRATAMENTO DA SÍNDROME METABÓLICA (I-DBSM). Sociedade Brasileira de Cardiologia. Arq. Bras. Cardiol., São Paulo, v. 84, supl. I, abr. 2005.

INSTITUTO DE MEDICINA (IOM). Dietary reference intakes for calcium and vitamin D. Nation Academ Press, Washington. 2011.

INTERNACIONAL DIABETES FEDERATION (IDF). The IDF consensus worldwide definition of the metabolic syndrome. Diabet. Med., Washington, 2006.

KAUR, J. A. Comprehensive review on metabolic syndrome. Cardiol. Res. Pract., India, v. 4, n. 6, p.1-22, Mar. 2014.

KAYANIYIL, S. et al. Association of 25(OH)D and PTH with metabolic syndrome and its traditional and nontraditional components. J. Clin. Endocrinol. Metabol., Springfield, v. 96, p. 168-175, Oct. 2011.

KHOSRAVI-BOROUJENI, D.; AHMED, F.; SARRAFZADEGAN, N. Is the association between vitamin $D$ and metabolic syndrome independent of other micronutrients. Int. J. Vitam. Nutr. Res., Austrália, v. 20, p. 1-16, July 2016

LEE, E. Y, et al. Association of metabolic syndrome and 25-hydroxyvitamin D with cognitive impairment among elderly Koreans. Geriatr. Gerontol. Int., Porto Rico, v. 20, n.6, p. 22-32, Sept. 2016.

LÉGER-GUIST'HAU, J. et al. Low socio-economic status is a newly identified independent risk factor for poor vitamin D status in severely obese adults. J. Hum. Nutr. Diet., Madrid, v. 15, p. 123-134, Mar. 2016.

LEVORATO, C. D. et al. Factors associated with the demand for health services from a gender-relational perspective. Ciênc. Saúde Coletiva, Ribeirão Preto, v. 19, n. 4, p. 1263-1274, Aug. 2014.

LOTUFO, P. A.; BENSENOR, I. J. M.. Raça e mortalidade cerebrovascular no Brasil. Rev. Saúde Pública, São Paulo, v. 47, n. 6, p.11-13, dez. 2013.

MAEDA, S.S. et al. Recomendações da Sociedade Brasileira de Endocrinologia e Metabologia (SBEM) para o diagnostic e tratamento da hipovitaminose D. Arq. Bras. Endrocrinol. Metab., São Paulo, v. 58, n. 5, p.411-433, 2014.

MALTA, D. C. et al. Evolução anual da prevalência de excesso de peso e obesidade em adultos nas capitais dos 26 estados brasileiros e no Distrito Federal entre 2006 e 2012. Rev Bras Epidemiol., Brasília, v. 60, n. 12, p. 267-278, 2014.
MARINHO, N. B. P. et al. Risk for type 2 diabetes mellitus and associated factors. Acta Paul. Enferm., Campinas, v. 26, n. 6, p. 569-574, Mar. 2013.

MAXIMINO, P. et al. Fatty acid intake and metabolic syndrome among overweight and obese women. Rev. Bras. Epidemiol., Brasília, v. 18, n. 4, 2015.

MIÑAMBRES, I. et al. The association of hypovitaminosis D with the metabolic syndrome is independent of the degree of obesity. Internat Schol Resea Networ Endocrinol., New York, v. 2012, p. 1-5, 2012.

NEVES, J. P. R. et al. Hipovitaminose D em idosos hipertensos. Arq. Bras. Endocrinol. Metab., São Paulo, v. 56-57, p. 415-422, 2012.

PINHEIRO, R. S. et al. Gênero, morbidade, acesso e utilização de serviços de saúde no Brasil. Ciênc. Saúde Coletiva, Rio de Janeiro, v. 7, n. 4, p. 687-707, Fev. 2002.

POZZAN, R. et al. Dislipidemia, síndrome metabólica e risco cardiovascular. Rev. SOCERJ, Rio de Janeiro, v.17, n.2, jan. 2004.

PREMAOR, M. O; FURLANETTO, T. W. Hipovitaminose D em adultos: entendendo melhor a apresentação de uma velha doença. Arq. Bras. Endocrinol. Metab., Porto Alegre, v. 50, n. 1, p. 25 - 37, fev. 2006.

RAFAELLI, R. A. et al. Influência da vitamina D nas doenças endocrinometabólicas. Semina: Ciências Biológicas e da Saúde, Londrina, v. 36, n. 1, p. 333-348, ago. 2015.

RIGO, J. C. et al. Prevalência de síndrome metabólica em idosos de uma comunidade: comparação entre três métodos diagnósticos. Arq. Bras. Cardiol., São Paulo, v.93, n.2, p.89-91, ago. 2009

SALEHPOUR, A. et al. A 12-week double-blind randomized clinical trial of vitamin D3 supplementation on body fat mass in healthy overweight and obese women. Nutr. J., Tehran, v.11, p. 78, Sept. 2012.

SANTOS JUNIOR, E. et al. Epidemiologia da deficiência de vitamina D. Revista Científica do ITPAC, Araguaína, v.4, n.3, p. 22-32, jun. 2011.

SANTOS, L. F.; ARAÚJO, E. M. Q.; MELO, J. B. Avaliação da prevalência de hipovitaminose $D$ em pacientes portadores de síndrome metabólica do centro de estudos e atendimento dietoterápico de Salvador, Bahia. Rev. Bras. Medic., [s.I], v.70, n. 7, 2013

SANTOS, A. Papel da vitamina D no risco cardiovascular. Rev Facto de Ris, Porto, v. 20, n. 9, p. 18-23, jun. 2011.

SANTOS, C.R.B. et al. Fatores dietéticos na prevenção e tratamento de comorbidades associadas à síndrome metabólica. Rev. Nutr., Campinas, v. 19, n. 3, p. 389-401, maio/jun. 2006.

SCHUCH, N. J; GARCIA, V. C; MARTINI, L. A. Vitamina D e doenças endocrinometabólicas. Arq. Bras. Endocrinol. Metab., São Paulo, v. 53, n.5, p. 625-633, 2009.

SILVA, A. et al. Prevalência de deficiência e insuficiência de vitamina De sua correlação com PTH, marcadores de remodelação óssea e densidade mineral óssea, em pacientes ambulatoriais. Arq. Bras. Endrocrinol. Metab., Belo Horizonte, v. 52, n. 3, p. 482-488, dez. 2008.

TEIXEIRA, A. C. et al. Prevalência de Síndrome metabólica em mulheres de 40 a 59 anos utilizando 2 critérios internacionais. Rev. Enferm. Cent. 0 .

Min., Belo Horizonte, v. 12, n. 4, p.1349-1358, set./dez. 2014.

VERRUSIO, D. R. et al. Association between serum vitamin D and metabolic syndrome in middle-aged and elderly adults and role of vitamin D supplementation therapy. Ann. Ist. Super Sanit., Madrid, v. 53, n.1, p.54-59, Dec. 2017. 
VIDIGAL, F. de C. et al. Prevalence of metabolic syndrome in Brazilian adults: a systematic review. BMC Public Health, Reus-Espanha, v. 5, n. 7, p. 49-51, Sept. 2013.

SOCIEDADE BRASILEIRA DE CARDIOLOGIA. VI-DSBC. VI Direrizes da Sociedade Brasileira de Hipertensão. Arq. Bras. Cardiol., São Paulo. 2010.

VIEIRA, E. C.; PEIXOTO, M. do R. G.; SILVEIRA, E. A. da. Prevalência e fatores associados à síndrome metabólica em idosos usuários do Sistema Único de Saúde. Rev. Bras. Epidemiol., Goiania, v. 17, n. 4, p. 805-817, out. /dez. 2014.

YIN, Y. et al. Correction of hypovitaminosis D does not improve the risk profile of the metabolic syndrome in a Chinese population: a randomized 1-year controlled trial. Ásia Pac. J. Clin. Nutr., Jinan-China, v. 25, n. 1, p. 71-7, 2016.

YIN, X. et al. Serum 25(OH)D is inversely associated with metabolic syndrome risk profile among urban middle-aged Chinese population. Nutr. J., Jinan-China, v. 11, n. 68, p. 1-7, 2012.

Submetido em: 11/10/2017

Aceito em: 10/11/2017 